\title{
CYP2C19-guided antiplatelet therapy: a cost-effectiveness analysis of 30-day and 1-year outcomes following percutaneous coronary intervention
}

\begin{abstract}
Aim: Determine whether using CYP2C19 genotype to optimize antiplatelet therapy selection is cost effective over the initial 30 days and 1-year following percutaneous coronary intervention. Materials \& methods: A cost-effectiveness analysis compared 30-day and 1-year outcomes and cost across three treatment strategies (universal clopidogrel, universal prasugrel, genotype-guided) in a hypothetical cohort. Results: Base-case scenario results at 30 days indicated that the incremental cost per major cardiovascular or bleeding event avoided for genotype-guided treatment was US\$8525 and US\$42,198 compared with universal clopidogrel and prasugrel, respectively. Probabilistic sensitivity analysis demonstrated that genotype-guided treatment was cost effective over 30 days and 1 year in 62 and $70 \%$ of simulations, respectively. Conclusion: Implementing a CYP2C19 genotype-guided approach to antiplatelet therapy could have a positive economic impact by preventing readmissions following percutaneous coronary intervention.
\end{abstract}

First draft submitted: 1 May 2017; Accepted for publication: 1 June 2017; Published online: 26 July 2017

Keywords: clopidogrel $\bullet$ cost-effectiveness $\bullet$ CYP2C19 $\bullet$ genotype $\bullet$ pharmacogenomics - prasugrel $\bullet$ readmission

Approximately 600,000 percutaneous coronary interventions (PCI) with intracoronary stent placement are performed annually in the USA in coronary artery disease (CAD) patients [1]. Dual antiplatelet therapy (DAPT) with aspirin and a P2Y $\mathrm{Y}_{12}$ inhibitor is indicated following PCI for stable CAD or an acute coronary syndrome (ACS) event [2-4]. Platelet activation and aggregation play a central role in the pathophysiology of CAD, leading to increased risk of major adverse cardiovascular events (MACE) including death, myocardial infarction, ischemic stroke and stent thrombosis (ST). DAPT is indicated to reduce the risk of thrombotic MACE and ST events, but increases the risk for major and minor bleeding events [4]. Selection of the $\mathrm{P} 2 \mathrm{Y}_{12}$ inhibitor is based on clinical factors, such as indication for PCI and risk factors for bleeding, and economic considerations.
In the USA, clopidogrel remains the most commonly prescribed P2 $\mathrm{Y}_{12}$ inhibitor $[5,6]$.

Clopidogrel is a prodrug that requires biotransformation by cytochrome P450 enzymes, specifically CYP2C19, to generate its active metabolite. Loss-of-function (LOF) polymorphisms in CYP2C19 are common and confer a reduced capacity for clopidogrel bioactivation and platelet inhibition [7]. Retrospective analyses of data from clinical trials and patient registries have demonstrated a higher risk for MACE and ST in clopidogrel-treated patients with one (intermediate metabolizer) or two (poor metabolizer) CYP2C19 LOF alleles after PCI compared with clopidogreltreated patients without an LOF allele (normal metabolizer) [8-10]. In contrast, CYP2C19 genotype does not alter the pharmacokinetics, antiplatelet effects or clinical response to prasugrel or ticagrelor $[10,11]$, which have shown
Mrudula S Borse', Olivia M Dong 2,3 , Melissa J Polasek², Joel F Farley', George A Stouffer ${ }^{4,5}$ \& Craig R Lee L $^{* 2,3,4}$ 'Division of Pharmaceutical Outcomes \& Policy, UNC Eshelman School of Pharmacy, University of North Carolina at Chapel Hill, Chapel Hill, NC 27599, USA ${ }^{2}$ Division of Pharmacotherapy \& Experimental Therapeutics, UNC Eshelman School of Pharmacy, University of North Carolina at Chapel Hill, Chapel Hill, NC 27599, USA

${ }^{3}$ UNC Center for Pharmacogenomics \& Individualized Therapy, University of North Carolina at Chapel Hill, Chapel Hill, NC 27599, USA

${ }^{4}$ UNC McAllister Heart Institute, University of North Carolina at Chapel Hill, Chapel Hill, NC 27599, USA

${ }^{5}$ Division of Cardiology, UNC School of Medicine, University of North Carolina at Chapel Hill, Chapel Hill, NC 27599, USA

*Author for correspondence:

Tel.: +19198437673

Fax: +1919962 0644

craig_lee@unc.edu 
superior efficacy compared with clopidogrel in ACS patients following PCI in clinical trials [12,13]. However, these alternative $\mathrm{P} 2 \mathrm{Y}_{12}$ inhibitors are more expensive than clopidogrel, which is available generically, and are associated with an increased bleeding risk [4,14-16].

Due to the clinical availability of CYP2C19 genotype testing, clinicians can now personalize antiplatelet therapy so the more potent and expensive alternatives to clopidogrel can be selectively prescribed in the subset of patients most likely to derive an inadequate response to clopidogrel [7]. However, there remains debate and uncertainty surrounding whether a CYP2C19 genotype-guided approach should be routinely used to optimize antiplatelet therapy selection following PCI as a means to improve patient outcomes and lower healthcare costs [17]. A number of recent studies have evaluated the cost-effectiveness of CYP2C19 genotyping for individualized antiplatelet therapy over 12-15 months of treatment [18-26]; however, the economic impact on the US healthcare system within the initial 30 days remains unclear. The Patient Protection and Affordable Care Act uses 'quality indicators' to adjust payments to hospitals as part of value-based purchasing [27]. Thirtyday hospital readmission rate is a proposed quality indicator for PCI by the National Quality Foundation; consequently, hospital readmissions within 30 days following PCI could lead to decreased reimbursements for health systems. Approximately $60-70 \%$ of MACE, ST and major bleeding events occur within the first 30 days following PCI [12-14,28-30]. These clinical events require readmission, confer significant morbidity and mortality to the patient, and in turn cause significant economic burden on the payer and the healthcare system. Therefore, identifying cost-effective strategies that improve the precision of $\mathrm{P}_{2} \mathrm{Y}_{12}$ inhibitor selection and minimize risk of major cardiovascular and bleeding events within 30 days of PCI is critical to healthcare systems, payers, providers and patients. The objective of the current study was to determine whether using a CYP2C19 genotype-guided strategy to optimize $\mathrm{P} 2 \mathrm{Y}_{12}$ inhibitor selection in CAD patients is cost effective over the initial 30 days and 1 year following PCI.

\section{Materials \& methods}

Model structure \& inputs

A decision tree model (Figure 1) was developed based on the US healthcare payer's perspective to compare three strategies for antiplatelet therapy in a hypothetical closed cohort of CAD patients undergoing PCI and treated with aspirin and a $\mathrm{P}_{2} \mathrm{Y}_{12}$ inhibitor for at least 12 months according to clinical practice guidelines [2,3]: universal clopidogrel (clopidogrel for all individuals without genotyping); universal prasugrel (prasugrel for all individuals without genotyping); and genotype-guided therapy (CYP2C19 genotyping with subsequent use of prasugrel for individuals carrying 1 or $2 \mathrm{LOF}$ alleles and clopidogrel for individuals with 0 LOF alleles). For patients undergoing CYP2C19 genetic testing, we assumed that all LOF allele carriers would receive prasugrel instead of clopidogrel, as recommended by the Clinical Pharmacogenetics Implementation Consortium guidelines [7]. It was also assumed that information on CYP2C19 genotype would be available either pre-emptively or with prompt turnaround to sufficiently guide drug selection during the index PCI hospitalization, as described by institutions that have implemented CYP2C19 genotyping into clinical practice [31-33]. Two time horizons were investigated with the same decision tree model: 30 days to evaluate early outcomes (primary analysis) and one year to evaluate total outcomes (secondary analysis).

Publicly available data sources and published studies were used for all model inputs. The prevalence of one or two CYP2C19 LOF alleles in the population was assumed to be $30 \%$, which is consistent with the frequency of the CYP2C19 intermediate or poor metabolizer phenotype in US populations [31,32]. The event rate probabilities for MACE (defined as composite of cardiovascular death, myocardial infarction or ischemic stroke events), ST (defined as definite or probable ST events according to the Academic Research Consortium criteria) and major bleeding (defined as major bleeding events unrelated to coronary artery bypass graft surgery according to the Thrombolysis in Myocardial Infarction [TIMI] criteria) at 30 days and 1 year were obtained from the metaanalysis by Mega and colleagues, which included nine studies of CAD patients undergoing PCI, with enrichment from the TRITON TIMI-38 clinical trial that compared clinical outcomes following randomization to either clopidogrel or prasugrel in ACS patients undergoing PCI [9,13]. The cost estimates used in the analysis are reported in 2014 US dollars (\$) and are based on Medicare reimbursement rates. The input parameters and assumptions used in the early outcome and total outcome analysis are summarized in Table 1, and are described in detail in the Supplementary Material.

\section{Analysis}

A cost-effectiveness analysis was conducted from the US healthcare payer's perspective.

In the model, a simulated cohort of 10,000 patients was assigned to each treatment strategy. The base-case scenario analysis assumed the base-case value for all parameters listed in Table 1. Model outcomes were calculated as the number of MACE, ST and major bleeding events avoided in the genotype-guided treatment arm compared with use of either universal clopidogrel or universal prasugrel per 10,000 patients treated. 


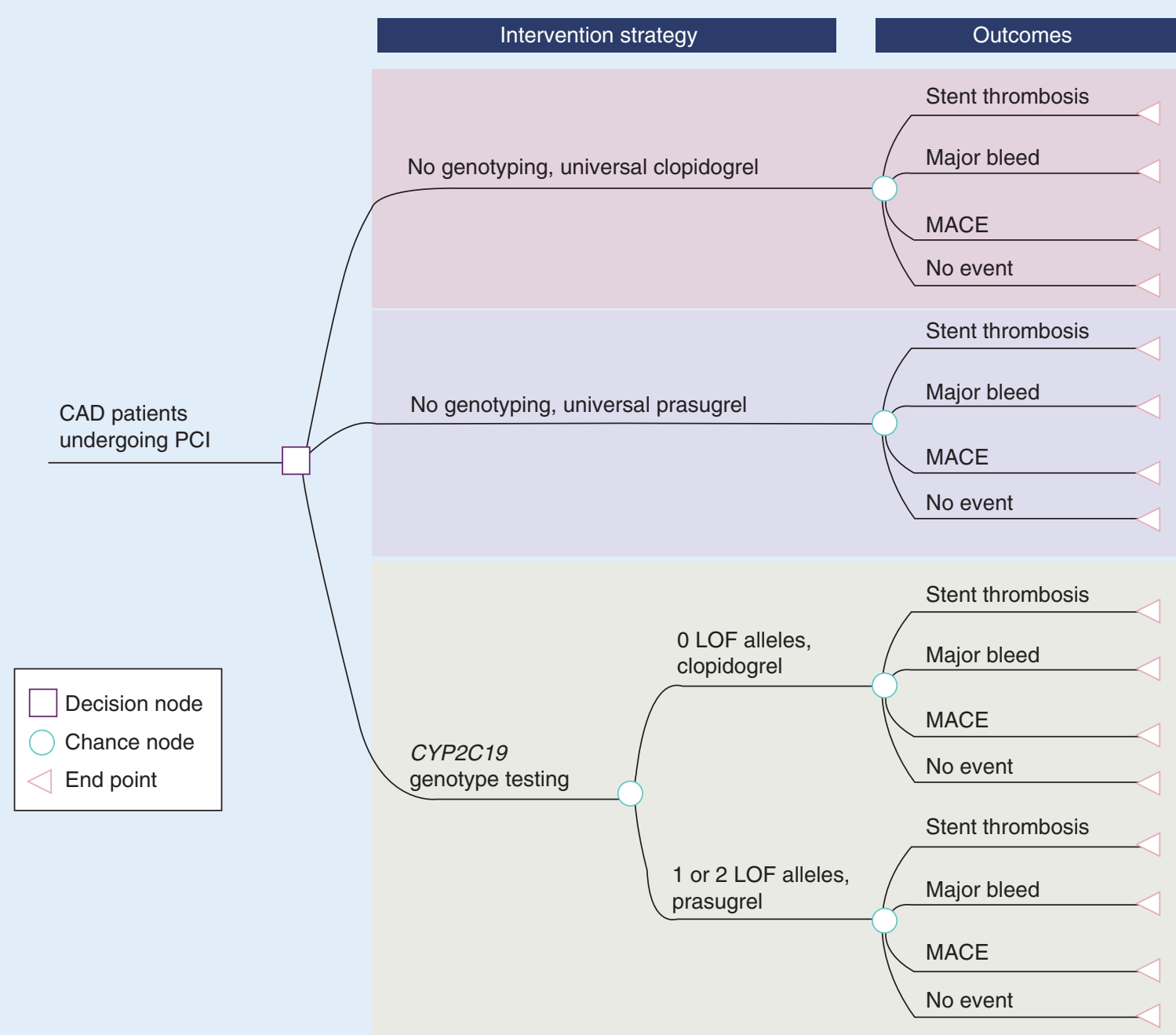

Figure 1. Model structure for the cost-effectiveness analysis. The structure of the decision tree is provided. Simulated patients enter the decision tree and proceed into one of the three intervention strategies: universal clopidogrel, universal prasugrel, or CYP2C19 genotype-guided treatment selection. Squares represent decision nodes, circles represent chance nodes and triangles represent terminal nodes.

CAD: Coronary artery disease; LOF: Loss-of-function; MACE: Major adverse cardiovascular events; PCI: Percutaneous coronary intervention.

The expected event costs per treated patient and the incremental cost-effectiveness ratio were also calculated. The incremental cost-effectiveness ratio value represents the cost to avoid a major cardiovascular or bleeding event.

Probabilistic sensitivity analyses were performed using 10,000 Monte Carlo simulations to investigate the impact of parameter uncertainty on the early outcome and total outcome cost-effectiveness results. All model inputs were parameterized using a triangular distribution with the base-case value representing the peak value and reported $95 \%$ confidence intervals or ranges, when available, or $\pm 20 \%$ of the base-case value representing the minimum and maximum values for individual input parameters (Table 1). All analyses were performed using TreeAge Pro 2016 (TreeAge Software, Inc., MA, USA). 
Table 1. Model input values used in the cost-effectiveness model.

\begin{tabular}{|c|c|c|c|}
\hline Parameter & Base-case value ${ }^{\dagger}$ & Lower limit ${ }^{*}$ & Upper limit ${ }^{ \pm}$ \\
\hline \multicolumn{4}{|l|}{ Costs (2014 US\$) } \\
\hline $\begin{array}{l}\text { Clopidogrel (generic) - } \\
\text { per 30-day supply }\end{array}$ & 13 & 6 & 23 \\
\hline $\begin{array}{l}\text { Prasugrel (brand) - per } \\
\text { 30-day supply }\end{array}$ & 324 & 260 & 390 \\
\hline CYP2C19 genotype test & 292 & 100 & 350 \\
\hline MACE & 8883 & 8715 & 16,611 \\
\hline ST event & 21,463 & 15,791 & 23,313 \\
\hline Major bleeding event & 8222 & 6623 & 11,258 \\
\hline \multicolumn{4}{|l|}{ Event rate probabilities } \\
\hline \multicolumn{4}{|c|}{ MACE on clopidogrel without genotype testing } \\
\hline Early (30 days) & 0.0610 & 0.055 & 0.070 \\
\hline Total (1 year) & 0.0960 & 0.089 & 0.106 \\
\hline \multicolumn{4}{|c|}{ MACE on clopidogrel with 0 LOF allele } \\
\hline Early (30 days) & 0.0500 & 0.042 & 0.057 \\
\hline Total (1 year) & 0.0820 & 0.070 & 0.089 \\
\hline \multicolumn{4}{|c|}{ MACE on prasugrel with 1-2 LOF allele or without genotype testing } \\
\hline Early (30 days) & 0.0500 & 0.042 & 0.057 \\
\hline Total (1 year) & 0.0820 & 0.070 & 0.089 \\
\hline \multicolumn{4}{|c|}{ ST on clopidogrel without genotype testing } \\
\hline Early (30 days) & 0.0132 & 0.010 & 0.016 \\
\hline Total (1 year) & 0.0195 & 0.014 & 0.024 \\
\hline \multicolumn{4}{|c|}{ ST on clopidogrel with 0 LOF allele } \\
\hline Early (30 days) & 0.0064 & 0.004 & 0.008 \\
\hline Total (1 year) & 0.0104 & 0.007 & 0.012 \\
\hline \multicolumn{4}{|c|}{ ST on prasugrel with 1-2 LOF allele or without genotype testing } \\
\hline Early (30 days) & 0.0064 & 0.004 & 0.008 \\
\hline Total ( 1 year) & 0.0104 & 0.007 & 0.012 \\
\hline \multicolumn{4}{|c|}{ Major bleed on clopidogrel without genotype testing } \\
\hline Early (30 days) & 0.0088 & 0.007 & 0.011 \\
\hline Total ( 1 year) & 0.0165 & 0.013 & 0.020 \\
\hline \multicolumn{4}{|c|}{ Major bleed on clopidogrel with 0 LOF allele } \\
\hline Early (30 days) & 0.0088 & 0.007 & 0.011 \\
\hline Total (1 year) & 0.0165 & 0.013 & 0.020 \\
\hline \multicolumn{4}{|c|}{ Major bleed on prasugrel with 1-2 LOF allele or without genotype testing } \\
\hline Early (30 days) & 0.0109 & 0.008 & 0.015 \\
\hline Total (1 year) & 0.0205 & 0.015 & 0.028 \\
\hline \multicolumn{4}{|c|}{ 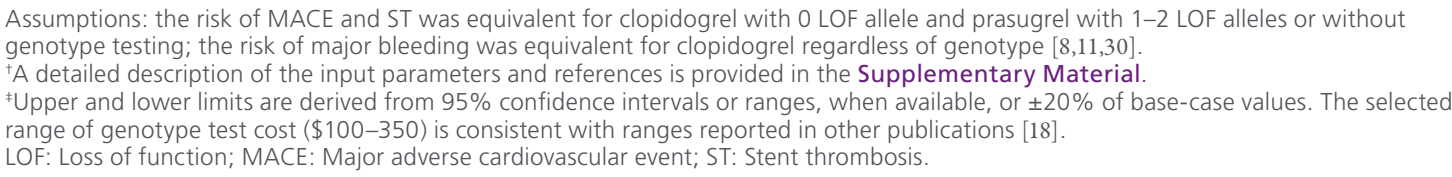 } \\
\hline
\end{tabular}


Table 1. Model input values used in the cost-effectiveness model (cont.).

\begin{tabular}{|c|c|c|c|}
\hline Parameter & Base-case value $^{\dagger}$ & Lower limit ${ }^{*}$ & Upper limit ${ }^{\ddagger}$ \\
\hline \multicolumn{4}{|c|}{ Other information (genetic test) } \\
\hline $\begin{array}{l}\text { Prevalence of } 0 \text { LOF } \\
\text { alleles in the population }\end{array}$ & 0.70 & 0.65 & 0.75 \\
\hline $\begin{array}{l}\text { Prevalence of } 1 \text { or } 2 \text { LOF } \\
\text { alleles in the population }\end{array}$ & 0.30 & 0.25 & 0.35 \\
\hline $\begin{array}{l}\text { Sensitivity of the genetic } \\
\text { test }\end{array}$ & 0.9999 & 0.95 & 1 \\
\hline $\begin{array}{l}\text { Specificity of the genetic } \\
\text { test }\end{array}$ & 0.9999 & 0.95 & 1 \\
\hline \multicolumn{4}{|c|}{$\begin{array}{l}\text { Assumptions: the risk of MACE and ST was equivalent for clopidogrel with } 0 \text { LOF allele and prasugrel with } 1-2 \text { LOF alleles or without } \\
\text { genotype testing; the risk of major bleeding was equivalent for clopidogrel regardless of genotype }[8,11,30] \text {. } \\
\text { tA detailed description of the input parameters and references is provided in the Supplementary Material. } \\
\text { tUpper and lower limits are derived from } 95 \% \text { confidence intervals or ranges, when available, or } \pm 20 \% \text { of base-case values. The selected } \\
\text { range of genotype test cost ( } \$ 100-350) \text { is consistent with ranges reported in other publications }[18] \text {. } \\
\text { LOF: Loss of function; MACE: Major adverse cardiovascular event; ST: Stent thrombosis. }\end{array}$} \\
\hline
\end{tabular}

\section{Results}

\section{Base-case scenario}

Early outcomes

When compared with universal clopidogrel, a genotype-guided treatment strategy was predicted to avoid 178 major cardiovascular events within 30 days (including 68 ST events) per 10,000 patients, while causing 6 major bleeding events, and would cost an additional US $\$ 147$ per patient (Tables 2 \& 3). When compared with universal prasugrel therapy, a genotypeguided strategy was predicted to prevent 15 major bleeding events within 30 days per 10,000 patients and cost an additional US\$62 per patient. Taking into account the cost of genetic testing, and assuming that all LOF allele carriers were treated with prasugrel, the incremental cost per major cardiovascular or bleeding event avoided for genotype-guided treatment was US\$8525 compared with universal clopidogrel and US $\$ 42,198$ compared with universal prasugrel, respectively (Table 3).

\section{Total outcomes}

When compared with universal clopidogrel, a genotype-guided treatment strategy was predicted to avoid 231 major cardiovascular events within 1 year (including 91 ST events) per 10,000 patients, while causing 12 major bleeding events, and would cost an additional US \$1102 per patient (Tables 4 \& 5). The incremental cost per major cardiovascular or bleeding event avoided for genotype-guided treatment was US $\$ 50,308$ compared with universal clopidogrel. When compared with universal prasugrel therapy without genotype testing, a genotype-guided strategy was dominant since it was predicted to prevent 28 major bleeding events within

Table 2. Base-case scenario results (early outcomes): number of major cardiovascular and bleeding events avoided over 30 days by genotype-guided therapy per 10,000 patients treated.

\begin{tabular}{|c|c|c|c|c|}
\hline Strategy & $\begin{array}{l}\text { MACE (composite) } \\
\text { events }^{\dagger}\end{array}$ & $\begin{array}{l}\text { Stent thrombosis } \\
\text { events }^{\ddagger}\end{array}$ & $\begin{array}{l}\text { Major bleeding } \\
\text { events }^{\S}\end{array}$ & $\begin{array}{l}\text { Total (sum) } \\
\text { events }\end{array}$ \\
\hline Universal clopidogrel & 610 & 132 & 88 & 830 \\
\hline Universal prasugrel & 500 & 64 & 109 & 673 \\
\hline Genotype-guided therapy & 500 & 64 & 94 & 658 \\
\hline $\begin{array}{l}\text { - Number of events avoided" } \\
\text { (vs universal clopidogrel) }\end{array}$ & 110 & 68 & -6 & 172 \\
\hline $\begin{array}{l}\text { - Number of events avoided" } \\
\text { (vs universal prasugrel) }\end{array}$ & 0 & 0 & 15 & 15 \\
\hline \multicolumn{5}{|c|}{$\begin{array}{l}{ }^{\dagger} \text { MACE defined as the composite of cardiovascular death, myocardial infarction or ischemic stroke events. } \\
\text { 'Defined as definite or probable stent thrombosis events according to the ARC criteria. } \\
\text { \$Defined as major bleeding events unrelated to coronary artery bypass graft surgery according to the TIMI criteria. } \\
\text { "A negative number indicates that the number of events in the genotype-guided therapy group is higher compared with the designated } \\
\text { reference group. }\end{array}$} \\
\hline
\end{tabular}




\begin{tabular}{|c|c|c|c|c|c|}
\hline Strategy & $\begin{array}{l}\text { Cost per } \\
\text { patient } \\
\text { (US\$) }\end{array}$ & $\begin{array}{l}\text { Incremental } \\
\text { cost (US\$) }\end{array}$ & $\begin{array}{l}\text { Adverse } \\
\text { events per } \\
\text { patient }\end{array}$ & $\begin{array}{l}\text { Incremental } \\
\text { effectiveness } \\
\text { (events avoided) }\end{array}$ & $\begin{array}{l}\text { Incremental cost- } \\
\text { effectiveness ratio } \\
\text { (cost per event } \\
\text { avoided, US\$) }\end{array}$ \\
\hline Universal clopidogrel & 910.53 & - & 0.0830 & - & - \\
\hline Universal prasugrel & 995.13 & & 0.0673 & & \\
\hline - vs universal clopidogrel ${ }^{\dagger}$ & & 84.60 & & 0.0157 & 5389 \\
\hline Genotype-guided therapy & 1057.16 & & 0.0658 & & \\
\hline - vs universal clopidogrel ${ }^{\dagger}$ & & 146.63 & & 0.0172 & 8525 \\
\hline - vs universal prasugrel ${ }^{\dagger}$ & & 62.03 & & $0.0015 w$ & 42,198 \\
\hline
\end{tabular}

1 year per 10,000 patients at a cost of US $\$ 2343$ less per patient.

\section{Probabilistic sensitivity analysis}

Probabilistic sensitivity analysis for early outcomes illustrated that genotype-guided treatment was predicted to be the most cost-effective strategy at a willingness-topay (WTP) threshold above US $\$ 12,000$, and genotype testing was increasingly cost effective compared with the other two options as the WTP threshold increased (Figure 2A). At a WTP threshold of US $\$ 50,000$, genotype testing was cost effective in $62 \%$ of the simulations, whereas prasugrel without genotype testing was cost effective in $38 \%$ of simulations. Universal clopidogrel was rarely cost effective over the initial 30 days. For total outcomes at 12 months, genotype-guided treatment was predicted to be the most cost-effective strategy at WTP thresholds above US $\$ 40,000$, and genotype testing was increasingly cost effective compared with the other two options as the WTP threshold increases
(Figure 2B). At a WTP threshold of US $\$ 50,000$, genotype-guided treatment was cost effective in $70 \%$ of the simulations, whereas universal clopidogrel was cost effective in $30 \%$ of simulations. Universal prasugrel was rarely cost effective due to the cost of prasugrel treatment over the course of 1 year.

\section{Discussion}

Implementation of a CYP2C19 genotype-guided approach to optimize antiplatelet therapy selection following PCI offers the potential to lower risk of major cardiovascular and bleeding events and lower healthcare costs [7]. However, the economic impact within the initial 30 days following PCI, when the risk for readmission due to a MACE, ST or bleeding event is highest, had not been evaluated. In the current investigation of a hypothetical US cohort of CAD patients undergoing PCI, selecting antiplatelet therapy based on a CYP2C19 genetic test result (LOF allele carrier: prasugrel, no LOF allele: clopidogrel)

\begin{tabular}{|c|c|c|c|c|}
\hline Strategy & $\begin{array}{l}\text { MACE } \\
\text { (composite) } \\
\text { events }^{\dagger}\end{array}$ & $\begin{array}{l}\text { Stent } \\
\text { thrombosis } \\
\text { events }^{\ddagger}\end{array}$ & $\begin{array}{l}\text { Major } \\
\text { bleeding } \\
\text { events }^{\S}\end{array}$ & $\begin{array}{l}\text { Total } \\
\text { (sum) } \\
\text { events }\end{array}$ \\
\hline Universal clopidogrel & 960 & 195 & 165 & 1320 \\
\hline Universal prasugrel & 820 & 104 & 205 & 1129 \\
\hline Genotype-guided therapy & 820 & 104 & 177 & 1101 \\
\hline - Number of events avoided" (vs universal clopidogrel) & 140 & 91 & -12 & 219 \\
\hline - Number of events avoided" (vs universal prasugrel) & 0 & 0 & 28 & 28 \\
\hline \multicolumn{5}{|c|}{$\begin{array}{l}\text { +MACE, defined as the composite of cardiovascular death, myocardial infarction or ischemic stroke events. } \\
\text { "Defined as definite or probable stent thrombosis events according to the ARC criteria. } \\
\text { "Defined as major bleeding events unrelated to coronary artery bypass graft surgery according to the TIMI criteria. } \\
\text { "A negative number indicates that the number of events in the genotype-guided therapy group is higher compared with the designated } \\
\text { reference group. } \\
\text { ARC: Academic Research Consortium; MACE: Major adverse cardiovascular event; TIMI: Thrombolysis in myocardial infarction. }\end{array}$} \\
\hline
\end{tabular}


Table 5. Base-case scenario results (total outcomes): cost- effectiveness of genotype-guided therapy over 1 year per patient treated.

\begin{tabular}{|c|c|c|c|c|c|}
\hline Strategy & $\begin{array}{l}\text { Cost per } \\
\text { patient } \\
\text { (US\$) }\end{array}$ & $\begin{array}{l}\text { Incremental } \\
\text { cost (US\$) }\end{array}$ & $\begin{array}{l}\text { Adverse } \\
\text { events per } \\
\text { patient }\end{array}$ & $\begin{array}{l}\text { Incremental } \\
\text { effectiveness } \\
\text { (events avoided) }\end{array}$ & $\begin{array}{l}\text { Incremental cost- } \\
\text { effectiveness ratio (cost } \\
\text { per event avoided, US\$) }\end{array}$ \\
\hline Universal clopidogrel & 1562.96 & - & 0.1320 & - & - \\
\hline Universal prasugrel & 5008.17 & & 0.1129 & & \\
\hline - vs universal clopidogrel ${ }^{\dagger}$ & & 3445.21 & & 0.0191 & 180,378 \\
\hline Genotype-guided therapy & 2664.70 & & 0.1101 & & \\
\hline - vs universal clopidogrel ${ }^{\dagger}$ & & 1101.74 & & 0.0219 & 50,308 \\
\hline - vs universal prasugrel ${ }^{\dagger}$ & & $-2343.47^{\ddagger}$ & & 0.0028 & 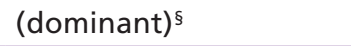 \\
\hline \multicolumn{6}{|c|}{$\begin{array}{l}{ }^{\dagger} \text { Reference group. } \\
{ }^{*} \text { A negative number indicates that the incremental cost in the genotype-guided therapy group is lower compared with the universal } \\
\text { prasugrel group. } \\
\text { §Universal prasugrel was predicted to be less effective and more expensive than (dominated by) genotype-guided therapy. }\end{array}$} \\
\hline
\end{tabular}

was projected to be more cost effective than either universal clopidogrel or universal prasugrel without CYP2C19 genotyping over the initial 30 days following PCI. Moreover, when considering outcomes and medication costs over 1 year, a genotype-guided strategy was predicted to be more effective and less costly than universal prasugrel as well as cost effective compared with universal clopidogrel. Taken together, these results suggest that routinely implementing a CYP2C19 genotype-guided approach to optimize $\mathrm{P} 2 \mathrm{Y}_{12}$ inhibitor use in clinical practice would have a positive economic impact on the US healthcare system.

Selection of medications that account for individual genetic differences has been proposed as a cornerstone of precision medicine [34]. As the evidence base grows and genetic tests are more readily available, clinicians have begun to implement genetic test results into treatment decisions for individual patients [35]. It is pertinent to understand the clinical and economic implications of genotype-guided prescribing on healthcare systems [36]. A series of previous costeffectiveness studies conducted in the US healthcare setting to understand the economic impact of CYP2C19 genotype testing to guide antiplatelet therapy selection following PCI have focused on longer term (12-15 months and lifetime) outcomes [18-24]. These studies have collectively concluded that CYP2C19 genotype-guided treatment strategies are cost effective when compared with universal clopidogrel or universal prasugrel treatment without genotype testing. Our 1-year results are consistent with these findings. However, it is equally important to understand the early economic impact of genotype testing, especially since there is a shift in the economic burden for hospital readmissions during the first 30 days following a PCI. Institutional protocol and the Center for Medicaid and Medicare Services mandates dictate that healthcare organizations could bear the burden of hospital readmissions in patients within the first 30 days of PCI compared with the later readmissions that are typically reimbursed by insurance.

The current investigation focuses on 30-day outcomes and costs, which has not been evaluated previously, and offers valuable new insight into the cost-effectiveness of a CYP2C19 genotype-guided treatment strategy during a critical time period when patients undergoing PCI are at highest risk for readmission due to adverse cardiovascular and bleeding outcomes. Base-case scenario results at 30 days indicated that genotype-guided therapy costs 16 and $7 \%$ more than universal clopidogrel and prasugrel, respectively, and is predicted to avoid MACE and ST events compared with universal clopidogrel and avoid major bleeding events compared with universal prasugrel. Consequently, the cost per major cardiovascular or bleeding event avoided for genotype-guided therapy was US $\$ 8525$ when compared with universal clopidogrel and US $\$ 42,198$ when compared with universal prasugrel. The base-case scenario and the probabilistic sensitivity analysis results indicated that a genotypeguided strategy is cost effective in the initial period after a PCI, and suggest that routine CYP2C19 testing might help healthcare institutions and payers save money by avoiding early readmissions after PCI. The projected cost savings to healthcare systems would be even more pronounced if reimbursement was reduced or eliminated due to hospitals being penalized for early readmissions.

When comparing the economic and clinical outcomes over 1 year to those observed at 30 days, the costeffectiveness of a genotype-guided strategy appeared to be lessened when compared with universal clopidogrel 


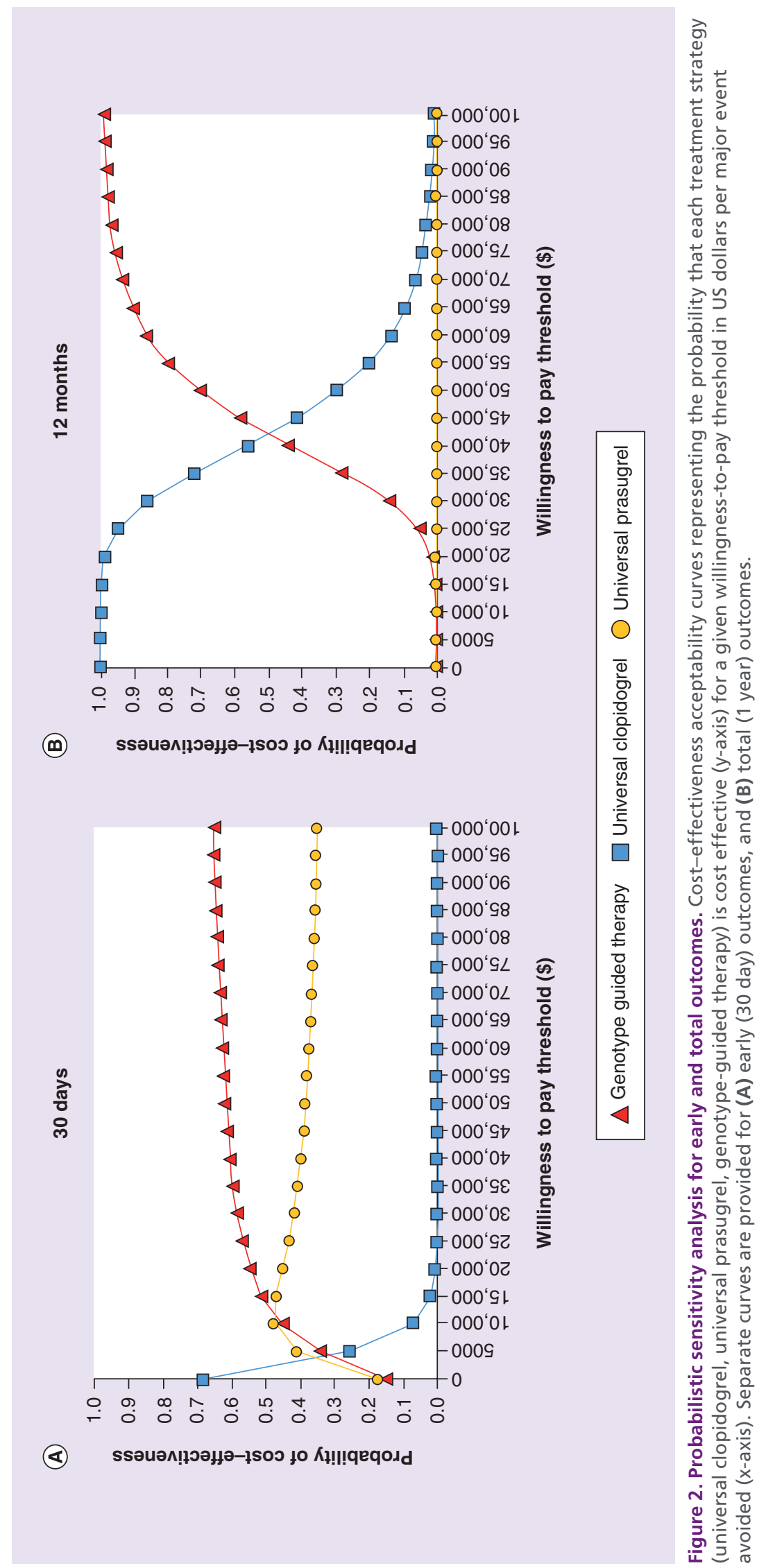


and magnified when compared with universal prasugrel. Genotype-guided therapy was predicted to cost $70 \%$ more than universal clopidogrel and cost $47 \%$ less than universal prasugrel therapy. These differences were driven by the cost of prasugrel treatment over the course of 1 year. Taking uncertainty across multiple input parameters in the probabilistic sensitivity analysis, genotype-guided treatment was predicted to be the most cost-effective strategy at a WTP threshold of US $\$ 50,000$ with cost-effectiveness predicted $62 \%$ of the time over 30 days and $70 \%$ of the time over 1 year. In comparison, universal prasugrel was predicted to be cost effective $38 \%$ of the time over 30 days and rarely over 1 year. Although universal prasugrel appears to be a cost-effective alternative to genotype-guided therapy over the first 30 days following PCI, the cost associated with prolonged prasugrel treatment made universal prasugrel the least cost effective option over the course of 1 year. Given that DAPT is indicated following PCI for at least 12 months in high risk patients $[2,3]$, and universal clopidogrel is associated with the highest risk of 30-day and 1-year MACE and ST events, universal genotyping with targeted use of prasugrel in CYP2C19 intermediate and poor metabolizers appears to be the preferred strategy in terms of cost-effectiveness in both the short- and long-term.

Although this study integrated critical parameter inputs and projected 30-day cost and effectiveness estimates for genotype-guided antiplatelet therapy for the first time, there are limitations to the current investigation that must be considered. First, projected event rate probabilities for genotype-guided therapy were derived from retrospective analyses of clinical trials and patient registries, and not an outcome-driven prospective randomized controlled trial. Although a clinical trial that will compare the impact of CYP2C19 genotypeguided antiplatelet therapy on MACE and bleeding outcomes to universal clopidogrel has been initiated (NCT01742117), it will not be completed until 2020. Second, only major cardiovascular and bleeding events resulting in readmission were taken into consideration in this analysis, and patients could only experience one event over each time horizon in the model. Compared with clopidogrel, it is well-established that prasugrel also lowers the risk of other ischemic events such as unstable angina and target vessel revascularization, and increases the risk of minor bleeding events [13]. Since these events are directly related to the intensity of antiplatelet therapy, the number of events avoided with genotype-guided therapy may have been underestimated. Third, the main outcome in this analysis, cost per major cardiovascular or major bleeding event avoided, should be interpreted with caution. Although this outcome has been used in previous analyses $[19,37]$, major cardiovascular and major bleeding events are the most clinically relevant outcomes leading to readmission following PCI, and a WTP threshold of US $\$ 50,000$ is generally considered cost effective for an additional quality-adjusted life year, a WTP threshold value for the cost of a healthcare technology to avoid a major cardiovascular or major bleeding event has not been established and each event could impact quality of life differently. Our model costs were based on the current Center for Medicaid and Medicare Services reimbursement rates, which may not be generalizable for all commercially insured plans. Furthermore, only direct inpatient medical costs associated with the major cardiovascular or bleeding event were considered. The cost-effectiveness of preventing these events may have been underestimated since these events incur additional downstream medical costs, such as followup outpatient encounters and chronic treatment, which were not considered. Moreover, since the true costs extend beyond the payer's perspective, future analyses from the societal perspective that include indirect (e.g., lost time from work), direct nonmedical (e.g., transportation) and intangible costs (e.g., pain and suffering) are needed.

It is also important to note that the decision tree was simplified with a few key assumptions that may not reflect real-world clinical practice. First, although ticagrelor is an alternative antiplatelet therapy that is not impacted by CYP2C19 genotype, our analysis focused on prasugrel since prasugrel is the most commonly prescribed alternative antiplatelet therapy at institutions that have implemented CYP2C19 genotyping into clinical practice [31-33]. Evaluating the cost-effectiveness of a genotype-guided strategy compared with universal ticagrelor over 30 days and 1 year was beyond the scope of the current analysis, and will require subsequent investigation. Furthermore, since the antiplatelet therapy prescribing decision will include factors beyond CYP2C19 genotype, such as risk for bleeding, our assumption that all CYP2C19 intermediate and poor metabolizers would receive prasugrel instead of clopidogrel may overestimate the benefits of the genotype-guided strategy. Indeed, early adopters of this strategy have reported that approximately $70-80 \%$ of LOF allele carriers have been prescribed alternative therapy in clinical practice [31,32]. It was also assumed that the CYP2C19 genotype result would be available to sufficiently guide drug selection during the index PCI hospitalization. This would require either a reactive genotyping approach with on-site genotyping and prompt turnaround or a pre-emptive genotyping approach, as described [31,32]. Consequently, successful implementation of a reactive genotyping approach may not be feasible at all institutions. If the genotyping test 
result was delayed, our results suggest that a universal prasugrel strategy during the first 30 days may be a cost-effective alternative to genotype-guided therapy that could be considered to minimize readmission for early MACE or ST events until the genotype result is available and the patient is beyond the high-risk 30-day period. An alternative approach would be to use a pre-emptive genotyping strategy, which would involve identifying and genotyping a larger patient cohort with the understanding that only a subset of genotyped patients will require a PCI, antiplatelet therapy and actionable use of the CYP2C19 result in the near future. The cost-effectiveness of pre-emptive genotyping strategies, which typically involve panelbased testing for multiple genetic markers, are not well-described in the literature and beyond the scope of the current analysis. Future analyses will be needed to ascertain the relative cost-effectiveness of reactive and pre-emptive approaches to genotype-guided antiplatelet therapy for health systems and payers.

\section{Conclusion}

In the current investigation of a hypothetical US cohort of CAD patients undergoing PCI, selecting antiplatelet therapy based on a CYP2C19 genetic test result was projected to avoid major cardiovascular or bleeding adverse events at reasonable costs over the course of 30 days and 1 year following PCI when compared with universal clopidogrel and universal prasugrel. Taken together, these results suggest that implementing a genotype-guided approach to optimize $\mathrm{P}_{2} \mathrm{Y}_{12}$ inhibitor selection in clinical practice could help health systems achieve lower readmission rates within the first 30 days following PCI, and that reimbursement for CYP2C19 genetic testing appears to be a worthwhile investment from the perspective of third party payers. Given the high potential for a positive economic impact on the US healthcare system, the direct effects of implementing a CYP2C19 genotype-guided selection strategy on clinical outcomes in real-world clinical practice, and the associated costs, should be evaluated.

\section{Financial \& competing interests disclosure}

The authors have no relevant affiliations or financial involvement with any organization or entity with a financial interest in or financial conflict with the subject matter or materials discussed in the manuscript. This includes employment, consultancies, honoraria, stock ownership or options, expert testimony, grants or patents received or pending, or royalties.

No writing assistance was utilized in the production of this manuscript.

\section{Supplementary data}

To view the supplementary data that accompany this paper please visit the journal website at: www.futuremedicine.com/ doi/full/10.2217/pgs-2017-0075

\section{Summary points}

\section{Background \& methods}

- There remains uncertainty surrounding whether CYP2C19 genotype should be routinely used to guide antiplatelet therapy selection following percutaneous coronary intervention ( $\mathrm{PCl})$.

- The current study evaluated the cost-effectiveness of a CYP2C19 genotype-guided strategy from the US healthcare payer's perspective.

- A decision tree model projected major cardiovascular and bleeding outcomes and cost across three treatment strategies (universal clopidogrel, universal prasugrel and genotype-guided therapy) over 30 days and 1 year following $\mathrm{PCl}$ in a hypothetical closed cohort of 10,000 PCI patients.

Results

- The base-case incremental cost per major cardiovascular or bleeding event avoided at 30 days was US $\$ 8525$ and US\$42,198 for a genotype-guided strategy compared with universal clopidogrel and prasugrel, respectively.

- Probabilistic sensitivity analysis demonstrated that genotype-guided treatment was cost-effective over 30 days and 1 year in 62 and $70 \%$ of simulations, respectively, at a willingness-to-pay threshold of US\$50,000.

Conclusion

- These results suggest that a CYP2C19 genotype-guided strategy to optimize antiplatelet therapy would have a positive economic impact on the healthcare system by lowering readmission rates for major cardiovascular and bleeding events within the first 30 days and 1 year following $\mathrm{PCl}$.

\section{References}

1 Dehmer GJ, Weaver D, Roe MT et al. A contemporary view of diagnostic cardiac catheterization and percutaneous coronary intervention in the United States: a report from the CathPCI Registry of the National Cardiovascular Data Registry, 2010 through June 2011. J. Am. Coll. Cardiol. 60(20), 2017-2031 (2012). 
2 Levine GN, Bates ER, Bittl JA et al. 2016 ACC/AHA guideline focused update on duration of dual antiplatelet therapy in patients with coronary artery disease: a report of the American College of Cardiology/American Heart Association Task Force on Clinical Practice Guidelines. J. Am. Coll. Cardiol. 68(10), 1082-1115 (2016).

3 Levine GN, Bates ER, Blankenship JC et al. 2011 ACCF/AHA/SCAI guideline for percutaneous coronary intervention: a report of the American College of Cardiology Foundation/American Heart Association Task Force on Practice Guidelines and the Society for Cardiovascular Angiography and Interventions. Circulation 124(23), e574-651 (2011).

4 Brilakis ES, Patel VG, Banerjee S. Medical management after coronary stent implantation: a review. JAMA 310(2), 189-198 (2013).

5 Fan W, Plent S, Prats J, Deliargyris EN. Trends in P2Y12 inhibitor use in patients referred for invasive evaluation of coronary artery disease in contemporary US practice. Am.J. Cardiol. 117(9), 1439-1443 (2016).

6 Sherwood MW, Wiviott SD, Peng SA et al. Early clopidogrel versus prasugrel use among contemporary STEMI and NSTEMI patients in the US: insights from the National Cardiovascular Data Registry. J. Am. Heart Assoc. 3(2), e000849 (2014).

7 Scott SA, Sangkuhl K, Stein CM et al. Clinical pharmacogenetics implementation consortium guidelines for CYP2C19 genotype and clopidogrel therapy: 2013 update. Clin. Pharmacol. Ther. 94(3), 317-323 (2013).

8 Mega JL, Close SL, Wiviott SD et al. Cytochrome P-450 polymorphisms and response to clopidogrel. N. Engl. J. Med. 360(4), 354-362 (2009).

9 Mega JL, Simon T, Collet JP et al. Reduced-function CYP2C19 genotype and risk of adverse clinical outcomes among patients treated with clopidogrel predominantly for PCI: a meta-analysis. JAMA 304(16), 1821-1830 (2010).

10 Wallentin L, James S, Storey RF et al. Effect of CYP2C19 and $A B C B 1$ single nucleotide polymorphisms on outcomes of treatment with ticagrelor versus clopidogrel for acute coronary syndromes: a genetic substudy of the PLATO trial. Lancet 376(9749), 1320-1328 (2010).

11 Mega JL, Close SL, Wiviott SD et al. Cytochrome P450 genetic polymorphisms and the response to prasugrel: relationship to pharmacokinetic, pharmacodynamic, and clinical outcomes. Circulation 119(19), 2553-2560 (2009).

12 Wallentin L, Becker RC, Budaj A et al. Ticagrelor versus clopidogrel in patients with acute coronary syndromes. N. Engl. J. Med. 361(11), 1045-1057 (2009).

13 Wiviott SD, Braunwald E, Mccabe $\mathrm{CH}$ et al. Prasugrel versus clopidogrel in patients with acute coronary syndromes. N. Engl. J. Med. 357(20), 2001-2015 (2007).

14 Hochholzer W, Wiviott SD, Antman EM et al. Predictors of bleeding and time dependence of association of bleeding with mortality: insights from the Trial to Assess Improvement in Therapeutic Outcomes by Optimizing Platelet Inhibition with Prasugrel-Thrombolysis in Myocardial Infarction 38 (TRITON-TIMI 38). Circulation 123(23), 2681-2689 (2011).
15 Wiviott SD, Desai N, Murphy SA et al. Efficacy and safety of intensive antiplatelet therapy with prasugrel from TRITONTIMI 38 in a core clinical cohort defined by worldwide regulatory agencies. Am. J. Cardiol. 108(7), 905-911 (2011).

16 Dinicolantonio JJ, D’ascenzo F, Tomek A, Chatterjee $S$, Niazi AK, Biondi-Zoccai G. Clopidogrel is safer than ticagrelor in regard to bleeds: a closer look at the PLATO trial. Int. J. Cardiol. 168(3), 1739-1744 (2013).

17 Holmes DR Jr., Dehmer GJ, Kaul S, Leifer D, O’Gara PT, Stein CM. ACCF/AHA clopidogrel clinical alert: approaches to the FDA 'boxed warning': a report of the American College of Cardiology Foundation Task Force on clinical expert consensus documents and the American Heart Association endorsed by the Society for Cardiovascular Angiography and Interventions and the Society of Thoracic Surgeons. J. Am. Coll. Cardiol. 56(4), 321-341 (2010).

18 Jiang M, You JH. Review of pharmacoeconomic evaluation of genotype-guided antiplatelet therapy. Expert Opin. Pharmacother. 16(5), 771-779 (2015).

19 Reese ES, Daniel Mullins C, Beitelshees AL, Onukwugha E. Cost-effectiveness of cytochrome P450 2C19 genotype screening for selection of antiplatelet therapy with clopidogrel or prasugrel. Pharmacotherapy 32(4), 323-332 (2012).

20 Guzauskas GF, Hughes DA, Bradley SM, Veenstra DL. A risk-benefit assessment of prasugrel, clopidogrel, and genotype-guided therapy in patients undergoing percutaneous coronary intervention. Clin. Pharmacol. Ther. 91(5), 829-837 (2012).

21 Lala A, Berger JS, Sharma G, Hochman JS, Scott Braithwaite $\mathrm{R}$, Ladapo JA. Genetic testing in patients with acute coronary syndrome undergoing percutaneous coronary intervention: a cost-effectiveness analysis. J. Thromb. Haemost. 11(1), 81-91 (2013).

22 Kazi DS, Garber AM, Shah RU et al. Cost-effectiveness of genotype-guided and dual antiplatelet therapies in acute coronary syndrome. Ann. Intern. Med. 160(4), 221-232 (2014).

23 Patel V, Lin FJ, Ojo O et al. Cost-utility analysis of genotypeguided antiplatelet therapy in patients with moderate-to-high risk acute coronary syndrome and planned percutaneous coronary intervention. Pharm. Pract. (Granada) 12(3), 438 (2014).

24 Johnson SG, Gruntowicz D, Chua T, Morlock RJ. Financial analysis of CYP2C19 genotyping in patients receiving dual antiplatelet therapy following acute coronary syndrome and percutaneous coronary intervention. J. Manag. Care Spec. Pharm. 21(7), 552-557 (2015).

25 Jiang M, You JH. Cost-effectiveness analysis of personalized antiplatelet therapy in patients with acute coronary syndrome. Pharmacogenomics 17(7), 701-713 (2016).

26 Wang Y, Yan BP, Liew D, Lee VW. Cost-effectiveness of cytochrome P450 2C19*2 genotype-guided selection of clopidogrel or ticagrelor in Chinese patients with acute coronary syndrome. Pharmacogenomics J. 240, 360-366 (2017).

27 Patient Protection Affordable Care Act Public Law 111-148. 111th Congress. Section 3025.

www.healthcare.gov/law/full/index.html 
28 Wiviott SD, Braunwald E, Mccabe $\mathrm{CH}$ et al. Intensive oral antiplatelet therapy for reduction of ischaemic events including stent thrombosis in patients with acute coronary syndromes treated with percutaneous coronary intervention and stenting in the TRITON-TIMI 38 trial: a subanalysis of a randomised trial. Lancet 371(9621), 1353-1363 (2008).

29 Quinlan DJ, Eikelboom JW, Goodman SG et al. Implications of variability in definition and reporting of major bleeding in randomized trials of oral P2Y12 inhibitors for acute coronary syndromes. Eur. Heart J. 32(18), 2256-2265 (2011).

30 Sorich MJ, Vitry A, Ward MB, Horowitz JD, Mckinnon RA. Prasugrel vs clopidogrel for cytochrome P450 2C19genotyped subgroups: integration of the TRITON-TIMI 38 trial data. J. Thromb. Haemost. 8(8), 1678-1684 (2010).

31 Lee JA, Lee CR, Reed BN et al. Implementation and evaluation of a CYP2C19 genotype-guided antiplatelet therapy algorithm in high-risk coronary artery disease patients. Pharmacogenomics 16(4), 303-313 (2015).

32 Weitzel KW, Elsey AR, Langaee TY et al. Clinical pharmacogenetics implementation: approaches, successes, and challenges. Am. J. Med. Genet. C Semin. Med. Genet. 166C(1), 56-67 (2014).

33 Pulley JM, Denny JC, Peterson JF et al. Operational implementation of prospective genotyping for personalized medicine: the design of the Vanderbilt PREDICT project. Clin. Pharmacol. Ther. 92(1), 87-95 (2012).

34 Relling MV, Evans WE. Pharmacogenomics in the clinic. Nature 526(7573), 343-350 (2015).

35 Dunnenberger HM, Crews KR, Hoffman JM et al. Preemptive clinical pharmacogenetics implementation: current programs in five US medical centers. Annu. Rev. Pharmacol. Toxicol. 55, 89-106 (2015).

36 Payne K, Shabaruddin FH. Cost-effectiveness analysis in pharmacogenomics. Pharmacogenomics 11(5), 643-646 (2010).

37 Straub N, Beivers A, Lenk E, Aradi D, Sibbing D. A model-based analysis of the clinical and economic impact of personalising P2Y12-receptor inhibition with platelet function testing in acute coronary syndrome patients. Thromb. Haemost. 111(2), 290-299 (2014). 